\title{
ANALYSIS OF MORTALITY IN PATIENTS ADMITTED IN MEDICAL INTENSIVE CARE UNIT OF KHAN RESEARCH LABORATORIES HOSPITAL, ISLAMABAD, PAKISTAN
}

\author{
Azmat Ali', Awais Saeed Abbasi ${ }^{2 \otimes}$, Fyza Saleem ${ }^{3}$
}

\begin{abstract}
OBJECTIVE: This study was conducted to ascertain the causes of death and mortality rate in patients admitted to medical intensive care unit (ICU) of Khan Research Laboratories (KRL) Hospital, Islamabad, Pakistan.

METHODS: Current study enrolled 775 patients admitted in Medical ICU of KRL Hospital, Islamabad, Pakistan from April 16, 2016 till April I6 2017. KRL Hospital is a 350 bedded hospital and has a I3-beded level II ICU. This was a descriptive study involving non-probability consecutive sampling. Statistical analysis was done using statistical package for social sciences (SPSS version 20).
\end{abstract}

RESULTS: Out of 775 patients, I 25 (16.1\%) patients died. Out of these 125 patients, $68(54.4 \%)$ were males and 57 (45.6\%) were females. Majority of patients $(n=69 ; 55.2 \%)$ were ranging in age from $7 \mathrm{I}-100$ years of age, while 37 $(29.6 \%)$ patients were ranging in age from $56-70$ years and 19 (I5.2\%) were less than 55 years of age. Among the deceased, pneumonia was the most common reason $(n=49 ; 39.2 \%)$ for admission followed by urinary tract infection $(n=16 ; 12.8 \%)$ and septicemia $(n=14 ; 11.2 \%)$. Hypertension $(n=33$; $26.4 \%$ ) was the most common co-morbid followed by diabetes mellitus $(n=24$; $19.2 \%)$ and ischemic heart disease $(n=18 ; 14.4 \%)$.

CONCLUSION: More than half of deceased patients admitted to ICU had age more than seventy years. Sepsis related conditions were responsible for death in more than sixty percent of patients. Majority of patients had diabetes mellitus, hypertension or ischemic heart disease as co-morbid conditions.

KEY WORDS: Intensive Care Unit (ICU) (MeSH); Mortality (MeSH); Sepsis (MeSH); Pneumonia (MeSH); Hypertension (MeSH); Diabetes Mellitus $(\mathrm{MeSH})$.

THIS ARTICLE MAY BE CITED AS: This article may be cited as: Ali A, Abbasi AS, Saleem F. Analysis of mortality in patients admitted in medical intensive care unit of Khan Research Laboratories Hospital, Islamabad, Pakistan. Khyber Med Univ J 20I8; I0(I):32-35.

\section{INTRODUCTION}

$\mathrm{A}^{\mathrm{c}}$ ccording to the census done in 998 total population of Islamabad was 805235 persons; Male 434239 (53.93\%) and Female 370996 (46.07 $\%)$.' The few intensive care unit (ICU) services that exist are often hampered by a shortage of bed capacities, physicians and nurses with specific training in intensive care, as well as material resources. $^{2,3}$ This often results in inadequate care associated with high mortality rates and adverse functional long-term outcomes. ${ }^{4}$ Management of critically ill patients requires significant human, infrastructural, and financial resources. These resources are typically limited in low-income countries. Major intensive care units (ICUs) are mostly found in large hospitals in urban or metropolitan areas. ${ }^{5}$ ICUs in highincome regions of the world have mortality rate between $1020.9 \% .^{6-8}$ In developing countries, due to insufficient early sepsis care and delayed presentation to hospital and subsequently to ICU, mortality from sepsis is high.' In developing countries ICU mortality is 40. $1 \% .{ }^{10}$ Sepsis and septic shock are the leading causes of mortality and morbidity in critical care units."
\ Department of Medicine, Khan Research Laboratory (KRL) Hospital, Islamabad, Pakistan.

Email:Ali99azmat@gmail.com

Tel: +92-32I53808I I

Date Submitted: June 8, 2017

Date Revised: January 30,2018

Date Accepted: February 6, 2018

In Asian countries, the overall hospital mortality is $44.5 \%$, and the ICU mortality is $36.7 \%$. The median length of stay in the ICU and hospital is 7 (with interquartile range [IQR] of 4-I2) days and I 8 (IQR I I-33) days, respectively, in survivors and 6 (IQR 3-I2) days and 10 (IQR 4-22) days, respectively, in nonsurvivors. ${ }^{12}$ Different predictive scoring systems are used in ICUs for measuring important outcomes typically mortality. For Acute Physiologic and Chronic Health Evaluation (APACHE), the predicted mortality is based upon the sum of categorical variables entered into a computer-generated algorithm. For Simplified Acute Physiologic Score (SAPS) and Mortality Prediction Model (MPM), the severity score, which is based upon the sum of the variables, is entered into an equation that calculates a predicted mortality. For Sequential Organ Failure Assessment (SOFA), sequential severity scores plot the trajectory of the clinical course to provide a semi-quantitative assessment of mortality, based upon multi-organ failure. However, clinicians should be aware of what the limitations are in a specific population of interest. As an example, SAPS 3 may underestimate mortality in patients with cancer and solid organ transplant, and SOFA may be more useful in a population with sepsis. ${ }^{13-16}$

As there is limited data regarding factors leading to mortality of patients in ICU settings from our region, this study was conducted to ascertain the causes of death and mortality rate in patients admitted to medical ICU of Khan Research Laboratories (KRL) Hospital, Islamabad, Pakistan.

\section{METHODS}

This study enrolled 775 patients admitted in Medical ICU of KRL Hospital, Islamabad, Pakistan from April 16, 2016 till April 16, 2017. This was a descriptive study involving non- 


\section{TABLE I: REASONS FOR ADMISSION OF DECEASED PATIENTS TO INTENSIVE CARE UNIT}

\begin{tabular}{|l|c|c|}
\hline Admission Diagnosis & $\begin{array}{c}\text { Frequency } \\
(\mathbf{n = 1 2 5})\end{array}$ & Percentage \\
\hline Pneumonia & 49 & 39.2 \\
\hline Urinary Tract Infection (UTI) & 16 & 12.8 \\
\hline Septicemia & 14 & $1 \mathrm{I} .2$ \\
\hline Cerebro-Vascular-Accident (CVA) & 10 & 08 \\
\hline Decompensated Chronic Liver Disease (DCLD) & 8 & 6.4 \\
\hline Acute Kidney Injury (AKI) & 5 & 04 \\
\hline Myocardial Infarction & 5 & 04 \\
\hline Hepatocellular Carcinoma (HCC) & 4 & 3.2 \\
\hline Left Ventricular Failure (LVF) & 4 & 3.2 \\
\hline Tuberculosis & 3 & 2.4 \\
\hline Diabetic Ketoacidosis (DKA) & 2 & 1.6 \\
\hline Upper Gastro-Intestinal Bleed & 2 & 1.6 \\
\hline Atrial Fibrillation & 2 & 1.6 \\
\hline Acute Leukemia & $\mathrm{I}$ & 0.8 \\
\hline
\end{tabular}

probability consecutive sampling, conducted after approval from ethical review board of the hospital. All deaths were analyzed using medical records. Age, gender, comorbid conditions and reason for admission were among the variables available for analysis.

KRL Hospital is a 350 bedded hospital and has a I3-bed ICU equipped with cardiac monitors, electronic beds, 3 ventilators, 2 isolation rooms and dialysis facility. It provides level II ICU, ${ }^{10}$ services to all kinds of critically ill patients. Level II care includes mechanical ventilation for longer than $24 \mathrm{~h}$, and specific organ support like dialysis and inotropic infusions. ${ }^{10}$ Hospital has a Medical Information System (MIS) department and all medical records are compiled and retained through computer based software.

Patients with trauma and surgery and those under 14 years of age were excluded from this study. Statistical Package for Social Sciences (SPSS) version 20 was used for data analysis. The clinical data of the study patients were stated as number of patients and percentages.

\section{RESULTS}

Out of 775 patients admitted in ICU, I 25 (I6.1\%) patients died during the stay in ICU. Out of these 125 patients, 68 (54.4\%) were males and 57 (45.6\%) were females. Majority of patients $(n=69 ; 55.2 \%)$ were ranging in age from $7 \mathrm{I}$ and 100 years of age, while 37 $(29.6 \%)$ patients were ranging in age from $56-70$ years and 19 (15.2\%) were less than 55 years of age.

Among the 125 patients who died, 65.6 $\%(n=82)$ patients had more than I reason for admission while $34.4 \%$ $(n=43)$ had single reason for admission. Pneumonia $39.2 \% \quad(n=49)$ was the most common reason for admission followed by UTI $12.8 \%(n=16)$ as shown in table I.

Table II illustrates the comorbid conditions among deceased patients. Hypertension ( $n=33 ; 26.4 \%)$ was the most common comorbid condition followed by diabetes mellitus $(n=24$; $19.2 \%)$ and ischemic heart disease $(n=18 ; 14.4 \%)$.

\section{DISCUSSION}

ICUs in most hospitals consume very high cost and sophisticated devices but mortality rates are still very high. Current study evaluated mainly the mortality along with some other parameters among patients admitted in ICU of a tertiary care facility of a developing country.

Our study found out that mortality rate among patients admitted to Medical ICU was $16.12 \%$. According to a study conducted in the Netherlands from 1997-200I, hospital mortality for ICUadmitted patients in the National Intensive Care Evaluation (NICE) registration was $12.9 \% .^{7}$ A 10 -year retrospective cohort study done using the Australian and New Zealand Intensive Care Society adult patient database found that overall mortality rate was $16.1 \% .{ }^{8}$ A retrospective audit conducted in a general ICU in a university hospital in Uganda from January 2003 to December 2009 found average mortality rate of $40.1 \%{ }^{6}$ Results of a I-month audit conducted in six hospitals in the Mersey region of the UK concluded that ICU mortality between medical referrals was $31.3 \% .^{17}$ Mortality in ICU patients is estimated to be about $15 \%$ in France. ${ }^{2}$

In a study conducted in Lahore, Medical ICU mortality was $17.8 \%$. $^{18}$ Current study is one of the largest study done to date in Pakistan and shows the mortality rate of $16.12 \%$ among Medical ICU patients. The reason for this as compared to other developing countries is early and effective referral system in our organization. It can also be attributed to better ICU facility including timely ventilator support, availability of newer generation

TABLE II: FREQUENCY OF COMORBID CONDITIONS AMONG DECEASED PATIENTS

\begin{tabular}{|l|c|c|}
\hline Co-Morbid & $\begin{array}{c}\text { Frequency } \\
(\mathbf{n = 1 2 5 )}\end{array}$ & Percentage \\
\hline Hypertension & 33 & 26.4 \\
\hline Diabetes Mellitus & 24 & 19.2 \\
\hline Ischemic Heart Disease & 18 & 14.4 \\
\hline Chronic Obstructive Pulmonary Disease & 18 & 14.4 \\
\hline Chronic Liver Disease & 15 & 12 \\
\hline Chronic Kidney Disease & 05 & 04 \\
\hline Pulmonary Tuberculosis & 01 & 0.8 \\
\hline Hepatocellular Carcinoma & 01 & 0.8 \\
\hline Breast Carcinoma & 01 & 0.8 \\
\hline Non-Hodgkin Lymphoma & 01 & 0.8 \\
\hline Lung Carcinoma & 01 & 0.8 \\
\hline None & 07 & 5.6 \\
\hline
\end{tabular}


antibiotics, trained and adequate number of nurses and paramedical staff.

Gender distribution among expired patients was $54.4 \%$ males versus $45.6 \%$ females. In a study conducted at Mulago hospital of Uganda majority of the patients $(56.5 \%)$ were males. ${ }^{10}$ In another study conducted in Lahore; among expired patients $56.6 \%$ were males while $43.4 \%$ were females. ${ }^{19}$ In our study majority of patients who died, $55.2 \%$ were between $7 \mathrm{I}$ and $I 00$ years. High mortality among elderly is because of the fact that multiple comorbid conditions exist in this age group.

In our study the most common diagnosis among patients who expired was Infection; including Pneumonia, Urinary Tract Infection, Pulmonary Tuberculosis and Septicemia. In a study conducted in Uganda, sepsis (49\%) was also the top most working diagnosis among expired patients. ${ }^{10}$ In another study done in China, sepsis was the most common diagnosis among expired patients. ${ }^{20}$ In developing countries, due to insufficient early sepsis care and delayed presentation to hospital and subsequently to ICU, mortality from sepsis is high.' High rate of infections among expired patients can also be attributed to the fact that majority of patients were elderly and had multiple comorbid conditions thus having low immunity and more prone to infections. Furthermore, lack of proper hygienic conditions and delayed presentation to hospital are major factors responsible for such high rates of infection. Due to traditional taboos, patients are taken to quacks first who obviously have no knowledge or skill to deal with such patients. Later they are taken to facilities which have paucity of resources (e.g. untrained staff, unavailability/unreliability of microbiological diagnostics and lack of appropriate antibiotics). After sufficient delay these patients are brought to tertiary care facilities but by then it had become almost impossible to save such patients as most of the times multi organ failure (MOF) has already occurred. Delayed and sometimes non availability of culture results renders the appropriate antibiotics choices difficult.

LIMITATION: This is a small scale, single-center study and results cannot be generalized to all centers in Pakistan. Blood and related culture results were not included in data analysis.

\section{CONCLUSION}

In our study, more than half of expired patients admitted to ICU had age more than seventy years. Sepsis related conditions were responsible for death in more than sixty percent of patients. Sixty percent of patients had diabetes mellitus, hypertension and ischemic heart disease as co-morbid conditions.

\section{REFERENCES}

I. Pakistan Bureau of Statistics, Government of Pakistan. Islamabad district at a glance. [Online]. [Cited on: May 30, 2017: ]. Available from URL: http://www.pbs.gov.pk/sites/ default/files//tables/District\%20at \%20glance\%20lslamabad.pdf

2. Baker T, Lugazia E, Eriksen J, Mwafongo V, Irestedt L, Konrad D. Emergency and critical care services in Tanzania: A survey of ten hospitals. BMC Health Serv Res 2013 Apr 16; I3: I40. DOI: I0. I |86/ |472-6963-13-|40.

3. Baelani I, Jochberger S, Laimer T, Otieno D, Kabutu J, Wilson I, et al. Availability of critical care resources to treat patients with severe sepsis or septic shock in Africa: a selfreported, continent-wide survey of anaesthesia providers. Crit Care 20II;I5:RI0. DOI:I0.II86/ cc94I0.

4. Frikha N, Mebazza M, Mnif L, El Euch N, Abassi M, Ben Ammar MS. Septic shock in a Tunisian intensive care unit: mortality and predictive factors. 100 cases. La Tunisie Medicale 0 I June 2005;83(6): 320325.

5. Dünser MW, Baelani I, Ganbold L. A review and analysis of intensive care medicine in the least developed countries. Crit Care Med 2006 Apr;34(4):I23442. DOI: I0.1097/ 0I.CCM.0000208360.70835.87.

6. McCrossan L, Bickerstaffe W, Mostafa SM, Anderson L, Cheater L, Jayson D, et al. Referrals to intensive care: A region-wide audit. Crit Care 2007; I I(I):403. DOI: I0.1।86/ cc5134.
7. De Jonge $\mathrm{E}$, Bosman RJ, van der Voort PH, Korsten HH, Scheffer GJ, de Keizer NF. Intensive care medicine in the Netherlands, $1997-$ 200 I. I. patient population and treatment outcome. Ned Tijdschr Ge neeskd $2003 \mathrm{May}$ 24; |47(21): 1013-7.

8. Moran JL, Bristow P, Solomon PJ, George C, Hart GK, Australian and New Zealand intensive care society database management committee (ADMC). Mortality and length-ofstay outcomes, 1993-2003, in the binational Australian and $\mathrm{New}$ Zealand intensive care adult patient database. Crit Care Med 2008 Jan;36(I):46-6I. DOI: I0.1097/ 0I.CCM.00002953। 3.08084.58.

9. Jacob ST, Moore CC, Banura P, Pinkerton R, Meya D, Opendi P, et al. Severe sepsis in two Ugandan hospitals: a prospective observational study of management and outcomes in a predominantly HIV-I infected population. PLoS One 2009 Nov II;4(I I):e7782. DOI: I0.137I/journal.pone. 0007782

10. Kwizera A, Dünser M, Nakibuuka J. National intensive care unit bed capacity and ICU patient characteristics in a low income country. BMC Res Notes 2012 Sep 1;5:475. DOI: I0.1 I86/I 756-0500-5-475.

I I. Qadeer A, Akhtar A, Qurat UI Ain, Saadat S, Mansoor S, Assad S, et al. Antibiogram of medical intensive care unit at tertiary care hospital setting of Pakistan. Cureus 2016 Sep; 8(9): e809. DOI: 10.7759/ cureus.809.

12. Phua J, Koh Y, Du B, Tang YQ, Divatia JV, Tan CC, et al. Management of severe sepsis in patients admitted to Asian intensive care units: prospective cohort study. BMJ 20II June I3; 342:d3245. DOI: 10.1 I36/bmj.d3245.

13. Costa e Silva VT, de Castro I, Liaño F, Muriel A, Rodríguez-Palomares JR, Yu L. Performance of the thirdgeneration models of severity scoring systems (APACHE IV, SAPS 3 and MPM-III) in acute kidney injury critically ill patients. Nephrol Dial Transplant 20II Dec;26(I2):3894- 
90I.DOI: 10.1093/ndt/gfr20I.

14. Soares M, Silva UV, Teles JM, Silva E, Caruso P, Lobo SM, et al. Validation of four prognostic scores in patients with cancer admitted to Brazilian intensive care units: results from a prospective multicenter study. Intensive Care Med 2010 July;36(7):। I88-95. DOI: 10.1007/ s00I34-0I0-1807-7.

15. Wu VC, Tsai HB, Yeh YC, Huang TM, Lin YF, Chou NK, et al. Patients supported by extracorporeal membrane oxygenation and acute dialysis: acute physiology and chronic health evaluation score in predicting hospital mortality. Artif Organs 2010 Oct;34(I0):828-35.

16. Maccariello E, Valente C, Nogueira L, Bonomo H, Ismael M, Machado
JE, et al. SAPS 3 scores at the start of renal replacement therapy predict mortality in critically ill patients with acute kidney injury. Kidney Int 2010 Jan;77(I):5I-6. DOI: 10.1038/ ki.2009.385.

17. McCrossan L, Bickerstaffe W, Mostafa SM, Anderson L, Cheater L, Jayson D, et al. Referrals to intensive care: A region-wide audit. Crit Care 2007;II(I): 403. DOI: I0.II86/ cc5134.

18. Shafiq F, ljaz AU, Kashif MA, Zafar S, Shahzad SI. Chronic liver disease related admissions and mortality pattern in medical ICU. Pak J Gastroenterol Mar 2006;20(I):72-4.

19. Shoukat H, Muhammad Y, Gondal KM, Aslam I. Mortality Prediction in Patients Admitted in Surgical
Intensive Care Unit by Using APACHE IV. J Coll Physicians Surg Pak Nov 20 I6;26(I I):877-80.

20. Zeng A, Song X, Dong J, Mitnitski A, Liu J, Guo Z, et al. Mortality in Relation to Frailty in Patients Admitted to a Specialized Geriatric Intensive Care Unit. J Gerontol A Biol Sci Med Sci 2015 Dec;70(I2): I586-94. DOI: 10.1093/gerona/glv084.

21. Azoulay E, Adrie C, De Lassence A, Pochard F, Moreau D, Thiery G, et al. Determinants of postintensive care unit mortality: a prospective multicenter study. Crit Care Med 2003 Feb;3I(2):428-32. DOI: I0.1097/0 I.CCM.0000048622.010 13.88.

\section{AUTHOR'S CONTRIBUTION}

Following authors have made substantial contributions to the manuscript as under:

AA: Concept \& study design, acquisition of data, drafting the manuscript, critical review, final approval of the version to be published

ASA \& FS: Acquisition, analysis \& interpretation of data, drafting the manuscript, final approval of the version to be published

Authors agree to be accountable for all aspects of the work in ensuring that questions related to the accuracy or integrity of any part of the work are appropriately investigated and resolved.

This is an Open Access article distributed under the terms of the Creative Commons Attribution-NonCommercial-NoDerivatives 4.0 International License (https://creativecommons.org/licenses/by-nc-nd/4.0/) which permits to reproduce freely in any medium and share the Licensed Material, for NonCommercial purposes only, provided the original work is properly cited. 